\title{
CONCEPTOS BÁSICOS DE PROGRAMACIÓN LINEAL Y APLICACIÓN EN EL MANEJO DE RECURSOS NATURALES
}

\section{Basic Concepts of Linear Programming and Application To Natural Resource Management}

\section{DANIEL HERNÁNDEZ-RAMÍREZ1', JORGE BLUHM-GUTIÉRREZ1, SANTIAGO VALLE-RODRÍGUEZ ${ }^{2}$}

${ }^{1}$ Hojalateros 14, Col. Tres Cruces, CP 98064, Zacatecas, Zac.

2Universidad Autónoma de Zacatecas Unidad Académica de Ciencias de la Tierra. Calzada Universidad \#108, Col. Progreso, CP 98064, Zacatecas, Zac. México.

E-mail: dhernan87@hotmail.com

\section{Resumen}

El presente es una revisión bibliográfica a profundidad que da a conocer grosso modo el concepto de Programación Lineal (PL), esto aplicándolo al uso, conocimiento y conservación de recursos naturales. Se analiza el origen de la técnica, los componentes básicos, ventajas y limitaciones del uso de PL en la gestión de recursos naturales, se analizaron así mismo algunas aplicaciones prácticas de la PL, se hizo un análisis de un caso y finalmente se concluye con base a los trabajos revisados que la PL es una herramienta metodológica que permite poder involucrar diversos factores relacionados con la gestión de recursos, es por eso que se vuelve un elemento primario por englobar tanto elementos ambientales, como sociales y económicos.

Palabras clave: Manejo, Recursos Naturales, Programación Lineal.

\begin{abstract}
This is a literature review in depth which discloses the concept of Linear Programming (PL) roughly, this applying to use knowledge and conservation of natural resources. The origin of the technique, the basic components, advantages and limitations of the use of PL in the management of natural resources is analyzed, some practical applications of PL were also analyzed, an analysis of a case is made and finally conclude based on It reviewed the work that PL is methodological tool that allows to involve several factors related to resource management, which is why it becomes a primary element encompass both environmental elements, such as social and economic.
\end{abstract}

Keywords: Management, Natural resources, Linear programming. 


\section{INTRODUCCIÓN}

Los recursos naturales ( $\mathrm{RN}$ ), se definen como un bien o servicio que surge de la naturaleza sin necesidad de intervención del ser humano. Resultan de vital importancia para el desarrollo de la sociedad humana (Campos et al. 2016), pues brindan la posibilidad de obtener aire, agua, alimentos, energía y en general elementos de subsistencia. El concepto involucra una gama muy amplia de opciones y posibilidades, además de interacciones que eventualmente, dificultan su aprovechamiento y conservación (PasosRippey 2010, Esteban et al. 2015). En este sentido, el uso y conservación de los RN se vuelven elementos estratégicos y de abordaje delicado, requieren de técnicas complejas que involucren la mayor cantidad de variables, tanto sociales, ambientales y económicas. La Programación Lineal (PL) es una propuesta concreta y directa para la mejor toma de decisiones en cuanto a manejo, uso y conservación de los RN (Guillen et al. 2004, Barrera 2016).

La PL es una metodología ampliamente utilizada con base en modelos matemáticos y en ocasiones complejos sistemas de ecuaciones. Se creó para dar un sentido práctico y de optimización de recursos en la búsqueda de obtener una mejor y más concreta solución, tal como sucede en la asignación y distribución de recursos cuando estos son limitados (Coronel \& Araujo 2004, Guillen et al. 2004). Surge como respuesta a la creciente necesidad de organización y toma de decisiones durante la Segunda Guerra Mundial, por lo que su naturaleza es de tipo estratégico-militar (Fauli \& Juan s/f (a); Coronel \& Araujo 2004). Se define como una técnica matemática empleada para la toma de decisiones con carácter científico (Fauli \& Juan s/f (a)), usada también en la planificación y manejo de tierras para la asignación óptima de recursos escasos (Bettelheim et al. 1965, Eden et al. 1990, Coronel \&Araujo 2004), esto mediante técnicas cuantitativas. En otras palabras, la PL es un método de optimización en el sentido de llegar invariablemente a lo más adecuado (Frank 2001, Fauli \& Juan s/f (a)).

Pretende resolver problemas y determinar la mejor combinación de actividades para optimizar recursos y se emplee sólo lo necesario (Coronel \& Araujo 2004), esta técnica está diseñada para apoyar a directivos en la planificación y toma de decisiones para la asignación de recursos (Guillen et al. 2004, Fauli \& Juan s/f (a)). Es el método más conocido de optimización, sus campos de acción son amplios y variados, van desde actividades militares, industriales, financieras, administrativas, de marketing, de trasporte, hasta el manejo y aprovechamiento de los RN; tal es el caso de actividades forestales maderables y no maderables (Bruce et al. 1989, Eden et al. 1990, Guillen et al. 2004, Fauli\& Juan s/f (a), Caballero \& Grossmann 2007,
López et al. 2014, Esteban et al. 2015). El progreso y la disponibilidad de computadoras, han hecho que la PL tenga un crecimiento acelerado y la hacen una de las herramientas más empleadas en la gestión de empresas (Coronel \& Araujo 2004, Caballero \& Grossmann 2007).

Con el presente trabajo se analizó la importancia del uso de herramientas metodológicas como la PL, aplicada al uso y conservación de los recursos naturales, se dan a conocer sus características, alcances y limitaciones metodológicas. Así mismo, se resalta la aplicabilidad del método para la toma de decisiones de los gestores de $\mathrm{RN}$, con un alto grado de objetividad y certeza en los resultados y sobre todo en el manejo de conflictos entre las partes involucradas.

\section{ORIGEN DE LA TÉCNICA DE PROGRAMACIÓN LINEAL}

La necesidad de planificar y organizar estuvo presente en los orígenes de las grandes civilizaciones humanas (Egipto, hace 4000 años antes de nuestra era), posteriormente en Grecia se elaboraron los primeros métodos de organización del trabajo y del tiempo, pero fue en tiempos de la Revolución Industrial en donde el interés por ordenar y optimizar los recursos se vieron ampliamente demandados, en función de mejorar y optimizar sistemas de producción, produciéndose un "caldo de cultivo" óptimo para el desarrollo de la técnica de PL (Caballero \& Grossmann 2007).

La PL no es una técnica reciente. Se aplicó por primera vez en la segunda Guerra Mundial para la solución de problemas con el trasporte y la dieta de los soldados. En 1947, Dantzig (2002) aplicó un método denominado como "Método Simplex", y fue utilizado para solucionar problemas de abastecimiento a la Fuerza Aérea Norteamericana, se dejó en claro que dicho método pudiera ser aplicado por las empresas para la toma de decisiones con carácter científico (Fauli \& Juan s/f (a), Cordonier 1973, Coronel \& Araujo 2004, Caballero \& Grossmann 2007). La aplicación de la PL en sistemas de empresas comenzó a partir de los años 50's, cuando economistas agrarios adoptan rápidamente el método pese a las dificultades técnicas, ya que el acceso a medios como las computadoras era complicado, por una parte requerían del equipo y por otra necesitaban capacitación extra en el uso de ordenadores. Es así como con el paso del tiempo y el desarrollo de nuevas tecnologías, se favoreció el uso de la PL (Coronel \& Araujo 2004).

La complejidad de los sistemas actuales de producción, ha tenido una marcada influencia en la manera de resolver 
problemas, conflictos y en la manera de proponer soluciones, cada vez hay más elementos a considerar para producir alimentos, para reducir y/o controlar fuentes contaminación. Día a día son situaciones que demandan atención urgente en relación a la creciente población humana (Martínez et al. 2002; Brotons 2011), siendo la PL una herramienta eficiente para la solución de algunos problemas de actualidad.

\section{COMPONENTES BÁSICOS DE LA PROGRAMACIÓN LINEAL}

De acuerdo con el concepto manejado por Coronel \& Araujo 2004, a la PL se le reconocen tres elementos básicos:

$\checkmark$ Función objetivo: es uno de los principales componentes, lo cual debe ser definido y encaminado claramente a resolver dos tipos de problemas, por una parte a maximizar un valor (ganancia, producción, espacio, ingreso, personal, beneficio, etc.) o bien minimizar un criterio (costos, insumos, contaminación, etc.).

$\checkmark$ Actividades posibles: corresponde a cada uno de los procesos posibles en el seno del sistema, pueden ser cultivos, producción de bienes, espacios laborales, acciones de conservación, venta de productos, número de especies, planes de manejo, tratamientos silvícolas, etc., las actividades deben de ser obligadamente más de una para tener sentido el uso de la PL, cuanto mayor sea el número de actividades y de alternativas, más útil será el método.

$\checkmark$ Restricciones: se refieren a que las actividades o alternativas se encuentran sujetas a limitaciones o restricciones dadas por las condiciones propias del sistema, son recursos en cantidades limitadas (tierra fértil, espacio, número de ejemplares, agua, etc.).

\section{VENTAJAS Y LIMITACIONES DE LA PROGRAMACIÓN LINEAL}

La PL para tener una funcionalidad, debe de considerar que los recursos están disponibles en cantidades limitadas. Se trata de un método de optimización, como ya se ha mencionado, se busca el mejor uso de los recursos en relación con la función objetivo. Si se dispone de cantidades ilimitadas de recursos para alcanzar el objetivo se hace redundante un uso racional de las materias (Guillen et al.
2004, Coronel \& Araujo 2004).En este sentido, una de las limitaciones en el uso de modelos matemático de PL, reside en su naturaleza metodológica, pues los modelos generados son normativos en un sentido que indica la mejor solución o la manera de optimizar los recursos (Coronel \& Araujo 2004).

En lo referente a las ventajas del uso de la PL, se mencionan algunas:

$\checkmark \quad$ Permite comparar un gran número de soluciones y alternativas en relativamente poco tiempo (Ramoset al. 2015).

$\checkmark \quad$ Indica a los tomadores de decisiones, como emplear eficazmente sus componentes en la búsqueda de optimizar recursos.

$\checkmark \quad$ Permite una toma de decisiones objetiva.

$\checkmark$ Reconoce "los cuellos de botella" y momento para corregirlos.

La labor más complicada y difícil de la PL, es reconocer y formular un problema a través de un modelo matemático (Frank 2001); es así como en lo referente a las limitaciones, se menciona lo siguiente:

$\checkmark \quad$ No genera expectativa de costos y precios.

$\checkmark \quad$ No contempla situaciones de riesgo, se basa en los supuestos de certeza de los datos.

$\checkmark \quad$ No es dinámico en cuanto a precios.

$\checkmark$ Requiere de abundante información para nutrir el sistema, entre más factores contemple, mayor será su efectividad.

Sin embargo, Coronel \& Araujo 2004, mencionan algunos problemas y sus soluciones parciales, refieren el uso de programas de cómputo adicionados con características que aminoren los inconvenientes.

\section{ALGUNAS APLICACIONES PRÁCTICAS DE LA PROGRAMACIÓN LINEAL}

El llevar a un mundo real es el reto más importante de la PL, no sólo son ecuaciones y fórmulas matemáticas, debe trascender a un plano práctico y aplicable a solucionar problemas tangibles surgidos en el seno de empresas, industrias y en este caso, el manejo, uso y aprovechamiento 
de los RN (Fauli \& Juan s/f (b)). En este sentido, la aplicación de la PL se vuelve amplia y diversa, entre las aplicaciones que se conoce es el área de salud (García et al. 2010), producción, investigación de mercados, marketing, logística, finanzas, empresariales, gestión (Fauli \& Juan s/f (b)) y puntualmente en el manejo y aprovechamiento de recursos forestales maderables y no maderables (Bravo et al. 1995, Vargas \& Ríos 2004, Díaz \& Prieto 1999, Galatsidas et al. 2013, González et al. 2013, López et al. 2014).

Para ser puntuales en los usos y aplicaciones documentadas de la PL, se menciona lo siguiente: a) La selección de medios publicitarios para determinar la combinación efectiva para anunciar productos; b) La delimitación de mercados al que debe de ir dirigido cierto producto; c) La manufactura de ropa y las proporciones de materiales en cada una de ellas; d) La planificación de la construcción de motores; e) El coste de producción y almacenamiento; f) La asignación de labores y distribución de tareas; g) La planificación y asignación de horarios en entidades comerciales; $h$ ) Minimizar costos por trasporte; e i) La optimización de dietas para pacientes en hospitales; entre otros (Fauli \& Juan $\mathrm{s} / \mathrm{f}(\mathrm{b})$ ). Así mismo, un ejemplo más en donde se ha aplicado la técnica de la PL es en la toma de decisiones referentes al uso, manejo y conservación de los RN (Guillen et al. 2004), ciertos casos serán abordados a continuación.

\section{ESTUDIO DE CASO: LA PROGRAMACIÓN LINEAL EN EL MANEJO DE LOS RECURSOS NATURALES}

El uso de los RN lleva consigo conflictos de intereses (Guillen et al. 2004), ya sea por excluir a ciertos grupos, por la percepción en ocasiones errónea de nuevos y contradictorios esquemas de manejo, por los desacuerdos al interior de las comunidades, e inclusive por desigualdades y carencia de políticas claras en el manejo y aprovechamiento, sin dejar de mencionar la influencia de actores externos (Matirú 2001, Gerritsen et al. 2014, Cortés et al. 2015).En este orden, los múltiples factores intrínsecos al uso, manejo y aprovechamiento de los RN son de consideración, ya que por el número de restricciones en aumento hacen compleja la toma de decisiones, dando origen a un ambiente difícil y en ocasiones comprometedor y hasta peligroso (Matirú 2001). Sin embargo, con el uso de herramientas metodológicas para la toma de decisiones como la PL, las decisiones toman un giro objetivo, además de plantear alternativas reales con un alto grado de cientificidad y optimización de recursos, tanto materiales como humanos (Guillen et al. 2004, Ramos et al. 2015).

Los trabajos analizados, todos relacionados con el aprovechamiento de los $\mathrm{RN}$, buscan abordar la ruta de la sustentabilidad y se encuentran con impedimentos de ciertas complejidades, sin embargo, todos reúnen abundantes factores a ser tomados en cuenta, tanto elementos sociales, económicos, culturales políticos, históricos y por supuesto ambientales y en cada uno de ellos se derivan otros, lo que hace complicada la toma de decisiones adecuadas y correctas (Guillen et al. 2004). En la figura 1, se aprecian, algunos ejemplos del uso de la PL para la toma de decisiones en el uso, manejo y aprovechamiento de los RN.

A continuación, se presenta un ejemplo de una situación que se puede resolver haciendo uso de la técnica de programación lineal (Marrero 1985):

Una dependencia de conservación de unidades naturales proporciona $1000 \mathrm{~m}^{3}$ de agua diarios para irrigar pinos $\mathrm{y}$ pasto. La superficie total disponible es de 15 ha. Los pinos requieren $80 \mathrm{~m}^{3} /$ ha por día y el pasto $40 \mathrm{~m}^{3} /$ ha diario. El pago por servicios ambientales es de $\$ 4000$ por ha de pinos y de $\$ 3000$ por ha de pasto. Se busca determinar la superficie que debe dedicarse a sembrar pinos y pasto, de tal manera que pueda obtenerse el máximo pago por servicios ambientales. El primer paso es determinar cuál es el objetivo, que en este caso es maximizar el beneficio:

$$
\begin{gathered}
X 1=\text { Superficie de pino } \\
\mathrm{X} 2=\text { Superficie de pasto } \\
\mathrm{MAXZ}=4000 \mathrm{X} 1+3000 \mathrm{X} 2
\end{gathered}
$$

$$
\text { Sujeta a: }
$$

$$
\begin{gathered}
\mathrm{X} 1+\mathrm{X} 2 \leq 15 \text { (Restricción } 1) \\
80 \mathrm{X} 1+40 \mathrm{X} 2 \leq 1000 \text { (Restricción } 2) \\
\text { Condiciones de no negatividad: } \\
\mathrm{X} 1 \geq 0 \\
\mathrm{X} 2 \geq 0
\end{gathered}
$$

En un problema bidimensional el espacio solución se define como un plano, con X1 medida a lo largo de la abscisa, y X2 a lo largo de la ordenada. Como las restricciones son lineales, se trazan sobre este plano como líneas rectas (Chapra \& Canale 2003). Al ser sólo dos variables, su solución puede efectuarse mediante procedimiento gráfico para resolverlo, construyendo una gráfica de dos dimensiones con X1 y X2 en los ejes (Hillier \& Lieberman 2006). Se representan las restricciones y la función objetivo como rectas, y se busca el máximo de la función objetivo planteado:

La solución encontrada es $X 1=5 / 3$ (superficie en hectáreas de pino), $X 2=40 / 3$ (superficie en hectáreas de pasto), y el máximo de la función objetivo es $Z=\$ 46666.67$ pesos por el 
Ambiente y Sostenibilidad 2016 (6): 97-104

Revista del Doctorado Interinstitucional en Ciencias Ambientales

ISSN:2339-3122

Digital

pago de servicios ambientales.

\section{MANEJO DE RECURSOS NATURALES (SUSTENTABILIDAD)}

\begin{tabular}{|c|}
\hline USO \\
\hline $\begin{array}{c}\text { Rehabilitación de suelo dañado } \\
\text { por actividades militares (Tucker } \\
\text { et al. 1998) }\end{array}$ \\
\hline
\end{tabular}

Costos de reducción de emisiones de $\mathrm{CO}_{2}$ (Petterson 2007)

Tratamiento de aguas residuales (Brotons 2011)

\begin{tabular}{|c|}
\hline $\begin{array}{c}\text { Uso y disposición de combustible } \\
\text { nuclear (Lee et al. 2012) }\end{array}$ \\
\hline $\begin{array}{c}\text { Turismo en Cuba y EUA (Pérez et } \\
\text { al. 2014; Valdivieso 2013) }\end{array}$ \\
\hline $\begin{array}{c}\text { Optimización de cargas de } \\
\text { nutrientes (Los et al. 2014) }\end{array}$ \\
\hline
\end{tabular}

$\mathrm{PL}$ en el sector forestal (Corel \& Araujo 2004)

Diseño de redes para reciclar agua (Zhao et al. 2016)

\begin{tabular}{|c|}
\hline MANEJO \\
\hline $\begin{array}{l}\text { Flujos de biomasa en ecosistemas } \\
\text { (Diffendorfer et al. 2001) }\end{array}$ \\
\hline $\begin{array}{l}\text { Valoración de los recursos } \\
\text { naturales (Guillen et al. 2004) }\end{array}$ \\
\hline $\begin{array}{c}\text { Análisis de cuencas y pago por } \\
\text { servicios ambientales (Estrada \& } \\
\text { Quintero 2004) }\end{array}$ \\
\hline $\begin{array}{l}\text { Modelo matemático para la } \\
\text { sustentabilidad (Ruiz 2011) }\end{array}$ \\
\hline $\begin{array}{l}\text { Ordenamiento del territorio } \\
\text { (Rodriguez \& Cubillos 2012) }\end{array}$ \\
\hline $\begin{array}{c}\text { Gestión de recursos forestales } \\
\text { (González et al. 2013) }\end{array}$ \\
\hline $\begin{array}{c}\text { Manejo de incendios (Rodríguez } \\
\text { \& Silva 2013) }\end{array}$ \\
\hline $\begin{array}{l}\text { Optimización de sistemas de } \\
\text { energía en edificios (Lauinger et } \\
\text { al. 2016; Ashouri et al. 2013) }\end{array}$ \\
\hline $\begin{array}{c}\text { Estrategias en creación de } \\
\text { parcelas forestales (Augustynczik } \\
\text { et al. 2016) }\end{array}$ \\
\hline
\end{tabular}

\section{APROVECHAMIENTO \\ Control de contaminantes \\ (Martinez et al. 2002) \\ Predicción en alternativas de manejo forestal (Gustafson et al. 2006)}

Ahorro de energía doméstica (Görkem \& Yükseltan 2012)

Optimización en extracción de energía geotermal (De Paly et al. 2012)

Aprovechamiento de leña (Gelatsidas et al. 2013)

Maximización en producción de azúcar, etanol y electricidad (Illukpitiya et al. 2013)

Producción azucarera (Blanco et al. 2014)

Suministros de mezcal en Zacatecas (López et al. 2014)

Asignación de recursos en compañia papelera (Wu et al. 2016)

Figura 1. Esquema general de algunas aplicaciones de la PL para el uso, manejo y aprovechamiento de RN. Fuente: Martínez et al. (2002), Coronel \& Araujo (2004), Guillen et al. (2004), Estrada \& Quintero (2004), Brotons (2011), Ruiz (2011), Rodríguez \& Cubillos (2012), Galatsidas et al. (2013), González et al. (2013), Rodríguez \& Silva (2013), Valdivieso (2013), Blanco et al. (2014), López et al. (2014), y Pérez et al. (2014).

\section{CONCLUSIONES}

La PL es una herramienta metodológica básica que facilita el trabajo interdisciplinario, su campo de acción es amplio, variado y complejo. Su poder de análisis es de consideración, no solo en industrias y empresas, también lo es para la toma de decisiones en el uso, conservación y manejo de los RN. Es evidente el avance de este tipo de análisis ante la exigencia de información cada vez más amplia y confiable, siendo esto una ventaja del método de PL.
La PL debe ser considerada como una orientación metodológica que apoya la toma de decisiones objetivas; para el caso del manejo de los $\mathrm{RN}$, en donde se busca optimizar los componentes del sistema en un esquema de sustentabilidad, su aplicación puede verse reflejada a diferentes escalas; por una parte, ofrece alternativas a grupos sociales reducidos (familias o comunidades), sin dejar de lado a grupos mayores como municipios, estados o regiones, e inclusive puede ser considerada para la toma de decisiones a escalas mayores (país), aludiendo al hecho de 
que a mayor escala, mayor complejidad y mayores son las dificultades para obtener información de todas las variables involucradas.

En este sentido, una limitante de la PL que reduce su operatividad, es la facilidad para disponer de información que nutra al sistema, mucha de esta información es de difícil acceso o simplemente no existe, debiéndose de generar, lo que trae por consecuencia el retraso del análisis; por otra parte, se sabe que entre más elementos contenga el análisis, más certeza tendrán los resultados.

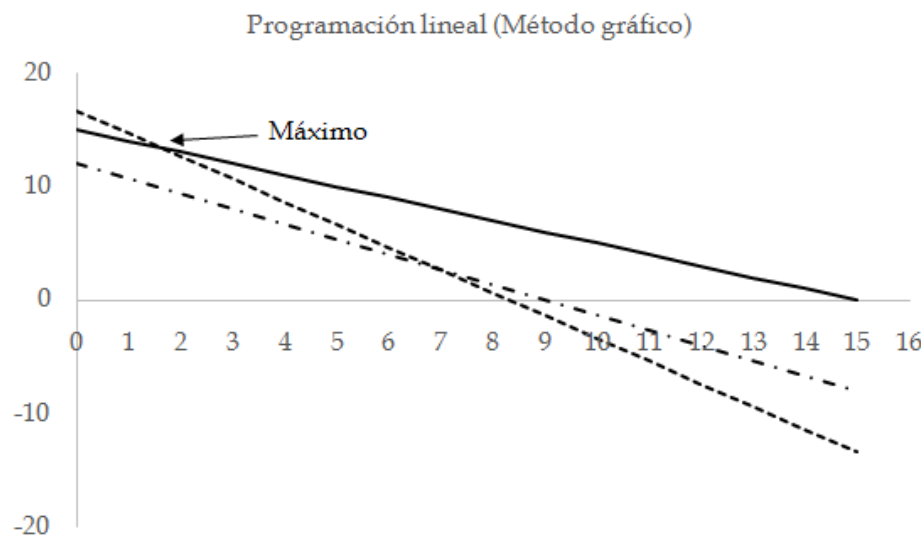

Figura 2. Solución gráfica de un problema de programación lineal enfocado al aprovechamiento de RN. Fuente: Elaboración propia.

El uso de instrumentos como la PL para el manejo, uso, conservación y aprovechamiento de los $\mathrm{RN}$, es una herramienta básica en la búsqueda de solución a conflictos entre los actores y problemas relacionados a la aplicación de esquemas de sustentabilidad en beneficio social, ambiental y económico.

\section{REFERENCIAS}

ASHOURI, A., FUX, S. S., BENZ, M. J., \& GUZZELLA, L. (2013). Optimal design and operation of building services using mixed-integer linear programming techniques. En: Energy 59: 365-376.

AUGUSTYNCZIK, A. L. D., ARCE, J. E. \& SILVA, A. C. L. (2016). Aggregating forest harvesting activities in forest plantations through Integer Linear Programming and Goal Programming. En: Journal of Forest Economics 24: 72-81.

BARRERA, V. H. (2016). Manejo de recursos naturales para agricultura de pequeña escala en la Ecoregión Andina de Ecuador.
BETTELHEIM, C., WEITZ, R., APPLEBAUM, L., KAHN, A. J., GIL BARRIOS, G. A., RODRÍGUEZ VARELA, A. N., ...\& TRIGO, E. J. (1965). Planificación y crecimiento acelerado (No. E10 191). IICA, La Paz (Bolivia).

BLANCO, N., ZUÑIGA C. A., ZUÑIGA, G. C., SIERRA, F. P., DIOS, P. R. \& CURBELO, A. A. J. (2014). Impacto del uso de la biomasa en la bioeconomía de los ingenios azucareros Centroamericanos. En: Revista científica de la UNAN- León. Vol. (5): 26-58.

BRAVO, F., RAMOS, M. T., ESTÉVEZ, A. R., \& AGUADO, J. S. (1995). Comparación del método del tramo móvil en regeneración con técnicas de programación lineal. Cuadernos de la Sociedad Española de Ciencias Forestales, (1).

BROTONS, M. J. M. (2011). La maximización del beneficio en las empresas depuradoras de aguas residuales. El caso de Valencia (España). En: Estudios Gerenciales, Vol. 27. No. 120 (jul-sep), págs. 147-165.

BRUCE, J. W., FORTMANN, L., LAWRY, S. W., YOUNG, J., SCHMINK, M., WOOD, C. H., \& HAYS, S. P. (1989). Agroforestry: tenure and incentives (No. GTZ-618). University of Wisconsin, Madison, Wis.(EUA). Land Tenure Center.

CABALLERO, J. A., \& GROSSMANN, I. E. (2007). Una revisión del estado del arte en optimización. En: Revista Iberoamericana de Automática e Informática Industrial. Vol. (4): págs. 5-23.

CAMPOS, F. J. A., CHÁVEZ, J. C. L. N., \& GARCÍA, V. M. G. (2016). El bienestar social en América Latina, 1990-2014: un análisis DEA a partir de las dimensiones del desarrollo humano. Revista Nicolaita de Estudios Económicos, 10(2), 728.

CHAPRA, S. C. \& CANALE, R. P. (2003). Métodos numéricos para ingenieros. McGraw-Hill Interamericana. D. F. México.

CORDONIER, P. (1973). Economía de la empresa agrícola. Ediciones Mundi-Prensa. Madrid. España.

CORTÉS, L. E. U. C., BARRERA, L. C., FERNÁNDEZ, M. A., \& MÉNDEZ, R. M. (2015). Manejo de los agroecosistemas en la comunidad lacandona de Nahá, Chiapas. Etnobiología, 11(3), 34-44.

CORONEL, R. M. \& ARAUJO, P. A. (2004) La Programación 
Ambiente y Sostenibilidad 2016 (6): 97-104

Revista del Doctorado Interinstitucional en Ciencias Ambientales

$\underset{\text { Digital }}{\text { ISSN:2339-3122 }}$

lineal aplicada al manejo forestal. Cátedra de Economía y Administración Forestal. Facultad de Ciencias Forestales de la Universidad Nacional de Santiago del Estero. Págs. 36.

DANTZIG, G. B. (2002). Linear Programming. En: Operations Research 50: 42-47.

DE PALY, M., HECHT-MÉNDEZ, J., BECK, M., BLUM, P., ZELL, A., \& BAYER, P. (2012). Optimization of energy extraction for closed shallow geothermal systems using linear programming. En: Geothermics 43: 57-65.

DÍAZ, B. L. \& PRIETO, R. (1999). Modelos de Planificación Forestal Basados en la Programación Lineal. Aplicación Al Monte "Pinar de Navafria" (Segovia). En: Invest. Agr.: Sist. Recur. For. Vol. 8 (1).

DIFFENDORFER, J. E., RICHARDS, P. M.; DALRYMPLE, G. H., \& DEANGELIS, D. L. (2001). Applying Linear Programming to estimate fluxes in ecosystems or food webs: an example from the herpetological assemblage of the freshwater Everglades. En: Ecological Modelling 144: 99-120.

EDEN, M. J., GARDNER, J. C., ANDERSON, V. L., SCHATZ, B. G., CARR, P. M., GULDAN, S. J., ... \& GOTTFRIED, R. (1990). Ecology and land management in Amazonia (No. GTZ-262). National Research Council, Washington, DC (EUA).

ESTEBAN, A. M., ZÁRATE, C. N., \& GRAÑA, F. M. (2015). Propuesta para el análisis de la competitividad de la terminal pesquera del puerto de Mar del Plata.

ESTRADA, D. \& QUINTERO, M. (2004). Propuesta metodológica para el análisis de cuenca: una alternativa para corregir las deficiencias detectadas en la implementación de pago por servicios ambientales. En: Electronic Forum on Payments Schemes. FAO and Redlanch. Págs. 11.

FAULI, J.\& JUAN, A. A. (s/f) a. Introducción a la investigación operativa. UOC. En: http://www.uoc.edu/in3/emath/docs/Intro_IO.pdf. Visto el 1 de mayo del 2015.

FAULI, J. \& JUAN, A. A. (s/f) b. Aplicación de la programación lineal. UOC. En: http://www.uoc.edu/in3/emath/docs/Aplicaciones_PL.p df. Visto el 2 de mayo de 2015.

FRANK, R. (2001). Planeamiento de la empresa con programación lineal. Documento de Administración Rural. Facultad de Agronomía. Universidad de Buenos Aires.

FAUBA. Buenos Aires.

GARCÍA R., J. F., GARCÍA, A., RODRÍGUEZ, G. \& GÁlVEZ, A. M. (2010). Herramientas de la evaluación económica y la investigación operacional que apoyan la toma de decisiones en salud. En: Salud en Tabasco, vol. 16, núm. 2-3, mayo-diciembre, pp. 933-938.

GALATSIDAS S., K. PETRIDISA, G. ARABATZISA, \& K. KONDOS. (2013). Forest production management and harvesting scheduling using dynamic Linear Programming (LP) models. En: Procedia Technology 8: 349-354.

GERRITSEN, P. R., MONTERO, M., \& FIGUEROA, P. (2014). El mundo en un espejo. Percepciones campesinas de los cambios ambientales en el occidente de México. Economía Sociedad y Territorio.

GONZÁLEZ, I., BLANCO E., HAYNES, J.A., BARRERO MEDEL, P., ÁLVAREZ, H., LAZO, D.A., CANDANO, A. F., EGAS F. A., VALDÉS E., LOUKOUNOZE, I., GAROFALO, N. A., ALAEJOS GUTIÉRREZ, M. C., GUERA OUOROU, J., GONZÁLEZ CRUZ, G. M.I., \& CARACIOLO FERREIRA, R.L.(2013). Aplicación de modelos ecológicos en la gestión de los recursos forestales en Cuba. En: J.A. Blanco (Ed.). Aplicaciones de modelos ecológicos a la gestión de recursos naturales. (pp. 141-180). Barcelona: Omnia Science.

GÖRKEM ÜÇTUG, F., \& YÜKSELTAN, E. (2012). A linear programming approach to household energy conservation: Efficient allocation of budget. En: Energy and Buidings 49: 200-208.

GUILLEN, R., FAUSTINO, J., VELÁZQUEZ, S., \& SOLÍS, H. (2004). Modelación del uso de la tierra para orientar el ordenamiento territorial en la subcuenca del rio Copán, Honduras. Consultado http://orton.catie.ac.cr/repdoc/A2187E/A2187E.PDF. Fecha: 4 de mayo del 2015.

GUSTAFSON, E. J., ROBERTS, L. J., \& LEEFERS, L. A. (2006). Linking linear programming and spatial simulation models to predict landscape effects of forest management alternatives. En: Journal of Environmental Management 81: 339-350.

HILLIER, F. S. \& LIEBERMAN, G. J. (2006). Introducción a la investigación de operaciones. McGraw-Hill Interamericana. D. F. México.

ILLUKPITIYA, P., YANAGIDA, J. F., OGOSHI, R., \& UEHARA, G. (2013). Sugar-ethanol-electricity co-generation in Hawai'i: An application of linear programming (LP) for 
optimizing strategies. En: Biomass and Bioenergy 48: 203212.

LAUINGER, D., CALIANDRO, P., VAN HERLE, J.,\& KUHN, D. (2016). A linear programming approach to the optimization of residential energy systems. En: Journal of Energy Storage 7: 24-37.

LEE, J., CHANG, Y. I., \& CHANG, S. H. (2012). Cost benefit analysis of advanced nuclear fuel cycle using Linear Programming optimization. En: Annals of Nuclear Energy: 116-120.

LÓPEZ, N. G.,MARTÍNEZ J. L., CAVAZOS, J.,\& MORENO, Y. M.(2014). La cadena de suministros del mezcal del estado de Zacatecas. En: Contaduría y Administración, 59 (2): 227252.

LOS, F. J., TROOST, T. A., \& VAN BEEK, J. K. L. (2014). Finding the optimal reduction to meet all targets-Applying Linear Programming with a nutrient tracer model of the North Sea. En: Journal of Marine Systems 131: 91-101.

MARRERO DE LEÓN, N. (1985). Técnicas de optimización aplicadas a la ingeniería hidráulica. ISPJAE. Ministerio de Educación Superior. La Habana. Cuba.

MARTÍNEZ, M. Y., UKA, S.,\& MURILLO, J. A.(2002). El control de la contaminación por nitratos en el regadío. En: Economía Agraria y Recursos Naturales. Vol. (2): 115-131.

MATIRU, V.(2001). Conflictos y manejo de recursos naturales. FAO, en: http://www.fao.org/forestry/2157509684b8bbf0673156ec237ead64c082b3.pdf. Visto el 2 de mayo de 2015.

PASOS RIPPEY, A.(2010). Perspectivas de la agricultura y del desarrollo rural en las Américas: Una mirada hacia America Latina y el Caribe. CEPAL. FAO. IICA.

PÉREZ, V., GUERRERO, F., GONZÁlEZ, M., PÉREZ, F.,\& CABALLERO, R.(2014). La sostenibilidad de los destinos cubanos de turismo de naturaleza: un enfoque cuantitativo. Tourism and Managment Studies. Vol. 10 (2): 32-40.

PETTERSSON, F. (2007). Carbon pricing and the diffusion of renewable power generation in Eastern Europe: A linear programming approach. En: Energy Policy 35: 2412-2425.

RAMOS, J. M., BRUNET, R. N., \& ALBELO, M. (2015). Modelo de programación lineal para optimizar alternativas agroproductivas en fincas del piedemonte amazónico. Momentos de Ciencia, 3(1).
RODRÍGUEZ, R. P. C. \& CUBILLOS, A.(2012). Elementos para la valoración integral de los recursos naturales: un puente entre la economía ambiental y la economía ecológica. En: Revista Gestión y Ambiente. Vol. 15 (1): 77-90.

RODRÍGUEZ Y SILVA, F. (2013). La predicción presupuestaria en la extinción de incendios forestales: una aproximación metodológica desde el registro de la experiencia y el análisis de la eficiencia. Edita: Sociedad de Ciencias Forestales en el $6^{\circ}$ Congreso Forestal Español del 10 al 14 de junio del 2013 en Vitoria, Gasteiz.

RUIZ, M. J.(2011). Un Modelo matemático para determinar la sustentabilidad de un bosque. En: Política y Cultura, Num. 36: 285-305.

TUCKER, J. L., RIDEOUT, D. B. \& SHAW R. B. (1998). Using linear programming to optimize rehabilitation and restoration of injured land: an application to US army training sites. En: Journal of Environmental Management 52: 173-182.

VARGAS, S. L. \& RÍOS-MERCADO, R. Z.(2004). Aplicación de la Programación Lineal en el sector forestal. En: Ingenierías, Julio-septiembre, Vol. VII, 24: 19-26.

VALDIVIESO, J. C.(2013). Evaluación de la capacidad de gestión eficiente de turismo en áreas protegidas. En: Smart Regions for a Smarter Growth Strategy: new challenges of the regional policy and potencials of cities to overcome a worldwide economic crisis. Palacio de convenciones y congresos de Oviedo, España, del 21 al 22 de noviembre.

WU, J., ZHU, Q., AN, Q., CHU, J., \& JI, X. (2016). Resource allocation based on context-dependent data envelopment analysis and a multi-objective linear programming approach. En: Computers \& Industrial Engineering 101: 8190.

ZHAO, H. P., WANG, Z. F., CHAN, T. C. \& LIU Z. Y. (2016). Design of regeneration recycling water networks by means of concentration potentials and a linear programming method. En: Journal of Cleaner Production 112: 4667-4673. 